\title{
Studies of the radiation environment on the Mars surface using the Geant4 toolkit
}

\author{
Jun-Liang Chen ${ }^{1}$ (D) Su-Jun Yun ${ }^{2}$ Tie-Kuang Dong ${ }^{3} \cdot$ Zhong-Zhou Ren $^{4} \cdot$ \\ Xiao-Ping Zhang ${ }^{5}$
}

Received: 20 July 2021/Revised: 19 November 2021/Accepted: 23 November 2021/Published online: 25 January 2022

(C) The Author(s) 2022

\begin{abstract}
The radiation environment on the surface of Mars is a potential threat for future manned exploration missions to this planet. In this study, a simple geometrical model was built for simulating the radiation environment on the Mars surface caused by galactic cosmic rays; the model was built and studied using the Geant 4 toolkit. The simulation results were compared with the data reported by a radiation assessment detector (RAD). The simulated spectra of neutrons, photons, protons, $\alpha$ particles, and
\end{abstract}

This work was supported by the National Natural Science Foundation of China (Nos. 12035011, 11535004, 11905103, 11947211, 11975167, 11761161001, 11565010, 11961141003, 11805103, $11673075,11303107,11120101005$, and 11235001), the National Key R\&D Program of China (Nos. 2018YFA0404403 and 2016YFE0129300), the Science and Technology Development Fund of Macau (No. 008/2017/AFJ), the Fundamental Research Funds for the Central Universities (Nos. 22120210138 and 22120200101), and by the China Post- doctoral Science Foundation (Nos. 2019M660095 and 2020T130478).

Jun-Liang Chen

chenjunliang_upc@163.com

Tie-Kuang Dong

tkdong@pmo.ac.cn

Zhong-Zhou Ren

zren@tongji.edu.cn

1 Key Laboratory of Modern Acoustics and Department of Physics, Nanjing University, Nanjing 210093, China

2 School of Electronic Engineering, Nanjing XiaoZhuang University, Hongjing Road, Nanjing 211171, China

3 Key Laboratory of Dark Matter and Space Astronomy, Purple Mountain Observatory, CAS, 2 West Beijing Rood, Nanjing 210008, China

4 School of Physics Science and Engineering, Tongji University, Siping Rood 1239, Shanghai 200092, China particle groups $Z=3-5, Z=6-8, Z=9-13$, and $Z=14$ 24 were in a reasonable agreement with the RAD data. However, for deuterons, tritons, and ${ }^{3} \mathrm{He}$, the simulations yielded much smaller values than for the corresponding RAD data. In addition, the particles' spectra within the $90^{\circ}$ zenith angle were also obtained. Based on these spectra, we calculated the radiation dose that would have been received by an average human body on Mars. The distribution of the dose throughout the human body was not uniform. The absorbed and equivalent doses for the brain were the highest among all of the organs, reaching $62.0 \pm 1.7 \mathrm{mGy} /$ $\mathrm{y}$ and $234.1 \pm 8.0 \mathrm{mSv} / \mathrm{y}$, respectively. The average absorbed and equivalent doses for the entire body were approximately $44 \mathrm{mGy} / \mathrm{y}$ and $153 \mathrm{mSv} / \mathrm{y}$, respectively. Further analysis revealed that most of the radiation dose was owing to $\alpha$ particles, protons, and heavy ions. We then studied the shielding effect of the Mars soil with respect to the radiation. The body dose decreased significantly with

State Key Laboratory of Lunar and Planetary Sciences, Macau University of Science and Technology,

Macao 999078, China 
increasing soil depth. At the depth of $1.5 \mathrm{~m}$, the effective dose for the entire body was $17.9 \pm 2.4 \mathrm{mSv} / \mathrm{y}$, lower than the dose limit for occupational exposure. At the depth of 3 $\mathrm{m}$, the effective dose to the body was $2.7 \pm 1.0 \mathrm{mSv} / \mathrm{y}$, still higher than the accepted dose limit.

Keywords Galactic cosmic rays - Radiation environment of the Mars · Absorbed dose - Equivalent dose - Geant4

\section{Introduction}

Space exploration has developed rapidly, and significant progress has been made in the past decades. Mars has become a hotspot for deep-space exploration missions. Mars exploration programs have been carried out, to learn about the evolution of Mars and for determining whether life exists on Mars [1]. The manned mission to Mars has also been on schedule [2]. A key factor that must be considered for manned space exploration missions is the space radiation, which is caused by galactic cosmic rays (GCRs) and solar particle events (SPEs) [3-5]. In the space, energetic particles can penetrate spacecraft and harm the instruments and astronauts [6]. Radiation-related health risks constitute a serious challenge for long-term manned space exploration missions [7, 8]. GCRs are mainly composed of charged nuclei, such as protons, $\alpha$ particles, and heavier nuclei with a broad range of energies, from sub$\mathrm{MeV}$ to hundreds of $\mathrm{TeV}[9,10]$. SPEs mainly consist of protons and $\alpha$ particles, with relatively lower energies compared with GCRs. Owing to the planet's thin atmospheric layer and the lack of a global magnetic field [11], the radiation intensity on Mars is much higher than that on Earth. Knowing the radiation environment on Mars and the radiation dose that is likely to be received by astronauts is very important for future manned missions to Mars $[11,12]$. This knowledge will allow decision-makers to accurately assess the corresponding health risks and is likely to provide a reference for designing radiation-protection gear $[13,14]$.

The radiation environment on Mars is complex and is defined by several factors. The shielding effect of the Mars atmosphere is much weaker than that of the Earth atmosphere. As a result, energetic particles in GCRs and SEPs can penetrate the Mars atmosphere, reaching the planet's surface. Owing to the irregularity and infrequency of SPEs, only GCRs were considered in this study. The interaction between GCRs and atmospheric molecules generates many secondary particles, which further interact with the planet's atmosphere, resulting in the complex radiation spectrum on the Mars surface. Albedo particles that are produced on the planet's surface also contribute to the radiation environment. The three main components of the radiation environment on the Mars surface were considered in this study. The Curiosity rover has carried out a long-term scientific exploration on Mars since it successfully landed on the planet in 2012. At the same time, an onboard radiation assessment detector (RAD) has been collecting radiation-related information on the Mars surface, including the particles' spectrum and the absorbed dose rate [15].

Some studies have shown that long-term exposure to the space radiation can harm humans [2]. Radiation can directly and indirectly affect DNA molecules in the human body, causing cell death or mutation and increasing the risk of cancer [16-18] and other diseases. The National Aeronautics and Space Administration (NASA) categorized the health risks owing to the space radiation into four groups: (1) degenerative tissue effects, (2) carcinogenesis, (3) acute and late central nervous system (CNS) effects, and (4) radiation-related syndromes [19]. Accurate calculation of the radiation dose received by astronauts is necessary for assessing the health risks associated with the space radiation. However, it is difficult to directly calculate the particles' spectra on the Mars surface and the associated radiation dose. Monte Carlo simulations can be used for addressing this problem. Some transport codes have been used for studying the radiation environment in the space and on Mars, such as HZETRN [20, 21], HETC-HEDS [22], FLUKA [23], PHITS [24], MCNP6 [25, 26] and Geant4 [27-30]. In Ref. [20-26], Slaba et al. studied the radiation environment on the Mars surface using these tools. Most of these studies have mainly focused on simulating the radiation environment on the Mars surface. Our work addresses two aspects of this research area. First, we used the G4QMD model to deal with the inelastic scattering of GCRs in the Mars atmosphere and soil, and for obtaining the particles' spectra on the Mars surface. Then, based on the simulated particles' spectra, we calculated the radiation dose received by a typical human body. To our knowledge, this is the first study attempting to estimate the radiation dosage to individual organs of a typical human on the Mars surface. The results and conclusions of this study can be used when planning future manned Mars exploration missions.

\section{The radiation environment on Mars}

\subsection{Model setup}

To reduce the simulation time, a simple geometrical model consisting of a cylinder (radius, $1 \mathrm{~km}$ ) was built, to represent the Martian environment. The cylinder was divided into 12 layers to represent the Martian regolith and atmosphere. The first layer from the bottom corresponded 
to the Martian regolith (thickness, $3 \mathrm{~m}$; density, $1.7 \mathrm{~g} / \mathrm{cm}^{3}$ ) [21]. The second layer was 4-m-thick, representing the atmospheric conditions on the Mars surface. From the third layer to the twelfth layer, each layer was 1-m-thick, and the column densities of the different layers were 11.820, 5.915, $2.624,1.069,0.395,0.131,0.035,0.009,0.003$, and 0.001 $\mathrm{g} / \mathrm{cm}^{2}$, respectively. The overall column density of these layers was approximately $22 \mathrm{~g} / \mathrm{cm}^{2}[21,31,32]$, representing the Mars atmosphere. The detector was a cylinder (radius, $1 \mathrm{~km}$; thickness, $3 \mathrm{~mm}$ ) and was positioned $1 \mathrm{~m}$ above the soil, for detecting and registering the Mars radiation environment. The planet's soil and atmosphere components were derived from Ref. [21] and are listed in Table 1. Primary GCRs were incident from the top of the geometrical structure, in the isotropic fashion. Once a particle passed through the detector, the information about the event was recorded, including the particle's type, its kinetic energy, and its angle of incidence.

The radiation environment simulations were performed using the Geant4-10.5.1 package (version10.5.1). This code is a track structure code that was developed at CERN for simulating the passage of particles through matter $[29,30]$. It provides many models that can be chosen by the user according to the specific application, and it has been widely used in high-energy physics, medical physics, and radiobiology. The physical parameters that were used for simulating the interaction between GCRs and the Mars medium are listed in Table 2. The Emstandard_opt3 physics list was used for describing the electromagnetic interactions of photons, leptons, hadrons, and ions, with energies in the $1 \mathrm{keV}-10 \mathrm{PeV}$ range [33]. The G4HadronPhysicsQGSP_BIC_HP physics list [34] was used for describing the inelastic scattering of nucleons and mesons with nuclei, for energies in the $0 \mathrm{eV}-100 \mathrm{TeV}$ range. In this physics list, the quark gluon string model (QGS) was used for describing high-energy hadronic interactions (12 GeV$100 \mathrm{TeV}$ range). The binary cascade model (BIC) was used for dealing with nucleon-nucleus interactions at intermediate energies, and high-precision neutron models (HPs) were used for dealing with neutron-nucleus interactions involving energies below $20 \mathrm{MeV}$. For the inelastic interactions of nuclei, the G4QMD model that was validated in our previous work was used [35]. In this model, all nucleons were considered as participant particles, and each

Table 1 Modeled soil and atmosphere components of Mars [21]

\begin{tabular}{ll}
\hline & Martian atmosphere \\
\hline Martian atmosphere & $\mathrm{CO}_{2}(95.7 \%), \mathrm{N}(2.7 \%), \mathrm{Ar}(1.6 \%)$ \\
Martian soil & $\mathrm{SiO}_{2}(51.2 \%), \mathrm{Fe}_{2} \mathrm{O}_{3}(9.3 \%), \mathrm{H}_{2} \mathrm{O}(7.4 \%)$ \\
& $\mathrm{Al}_{2} \mathrm{CaK}_{2} \mathrm{MgNa}_{2} \mathrm{O}_{7}(32.1 \%)$ \\
\hline
\end{tabular}

Table 2 The physics list

\begin{tabular}{ll}
\hline Models & Description \\
\hline Emstandard_opt3 & Electromagnetic model \\
G4HadronPhysicQGSP_BIC_HP & Hadronic inelastic model \\
G4IonQMDPhysics & Hadronic inelastic model for ions \\
\hline
\end{tabular}

nucleon was treated as a Gaussian wave packet. Equations of the particles' motion were derived based on the time-dependent variation principle [36]. A detailed description of these models can be found in the Geant 4 Physics Reference Manual [36].

In this study, only GCRs were considered as contributing to the radiation environment of Mars. GCRs mainly consist of protons, $\alpha$ particles, and heavy ions. Although the proportion of heavy particles is very small, they are important for radiation protection, owing to their high relative biological effectiveness (RBE). In our simulations, particles with atomic numbers $Z=1-26$ were considered for GCRs. The energy spectra of these particles were calculated using the GCR model developed by Matthi $\ddot{a}$ et al. [10]. In that model, a single parameter $W$ was used for describing the modulation effect of solar activity [10]. A part of the primary GCR spectra used in the simulation is shown in Fig. 1. The modulation parameter $W$ was calculated according to the Oulu neutron monitor count rate (from http://cosmicrays.oulu.fi/) during the period from August 2012 to January 2013, and an average value $(W=56)$ was used in this work.

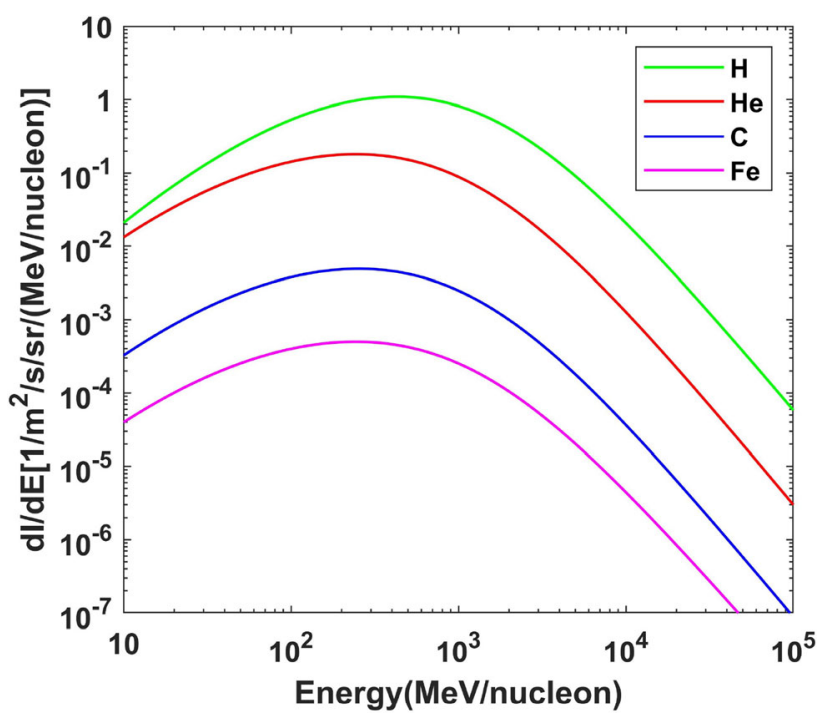

Fig. 1 (Color online) A part of the primary GCR spectra used in the simulation 


\subsection{Results}

When GCRs pass through a planet's atmosphere, many neutrons and photons are induced owing to the interactions between the GCRs and atmospheric molecules. The simulated neutron and photon spectra on the Mars surface are plotted in Fig. 2, together with the experimental data $[21,37]$. As shown in this figure, the simulation results for neutrons were slightly higher than the corresponding experimental data, for energies below $100 \mathrm{MeV} / \mathrm{n}$, while the simulation results for photons agreed well with the RAD data. For neutrons and photons, the average relative deviations of the simulation results from the corresponding experimental data were $60.8 \%$ and $56.0 \%$, respectively.

The simulation results for the charged particles' spectra, for zenith angles smaller than $30^{\circ}$, are plotted in Figs. 3 and 4. Heavy particles can be divided into several groups, for proper comparison to the RAD data [15]. Evidently, the simulated spectra of protons, $\alpha$ particles, and particle groups $Z=6-9, Z=9-13$, and $Z=14-24$ were consistent with the corresponding RAD data, and the corresponding average relative deviations were $27.7 \%, 37.9 \%$, $40.9 \%, 60.7 \%$, and $40.1 \%$. For the particle groups $Z=$ $3-5$ and $Z>24$, the simulation results were slightly lower than the measured data. However, the simulated spectra of deuterons, tritons, and ${ }^{3} \mathrm{He}$ were much lower than the corresponding RAD data, and the average relative deviation was approximately $90 \%$. These particles are pure secondary particles generated in reactions between primary GCRs and atmospheric molecules, and the observed discrepancy might be owing to the lower generation cross

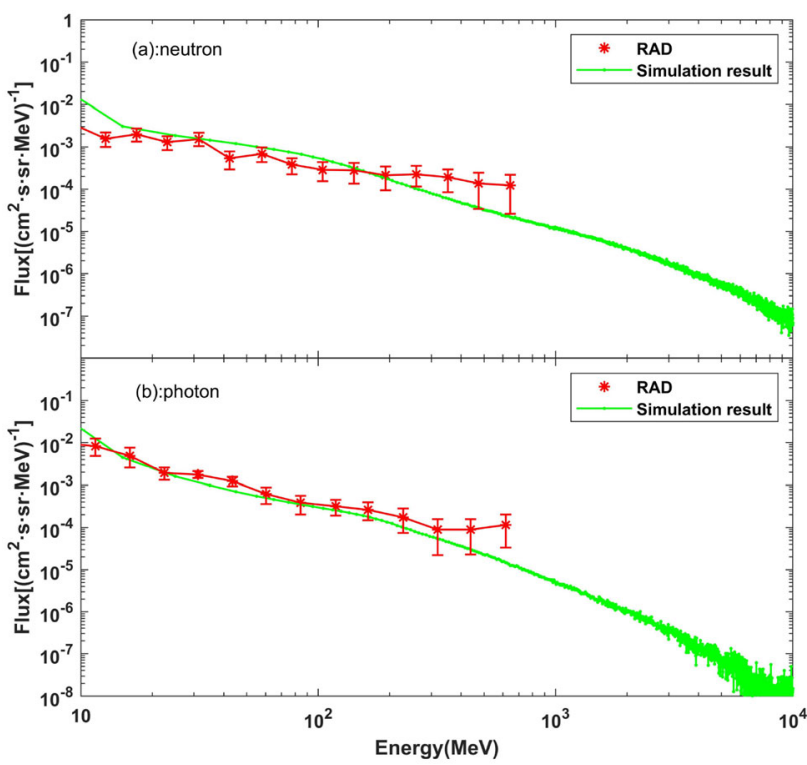

Fig. 2 (Color online) Simulated and measured photon and neutron spectra, for the Mars surface

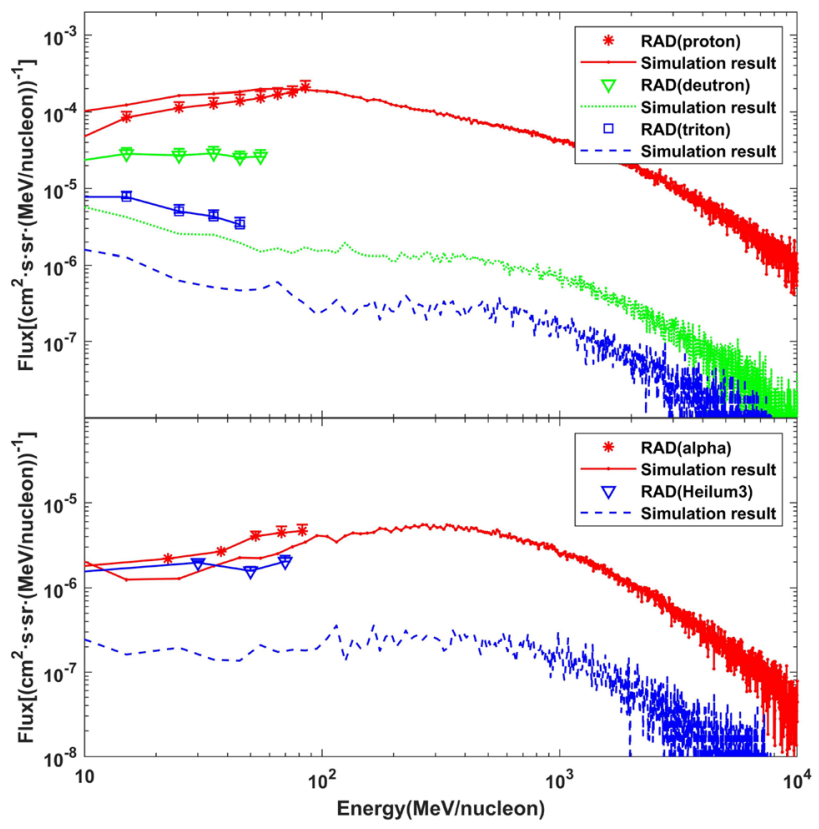

Fig. 3 (Color online) Simulated and measured charged particles' spectra, for zenith angles smaller than $30^{\circ}$ : a protons, deuterons, and tritons, b alpha particles and ${ }^{3} \mathrm{He}$

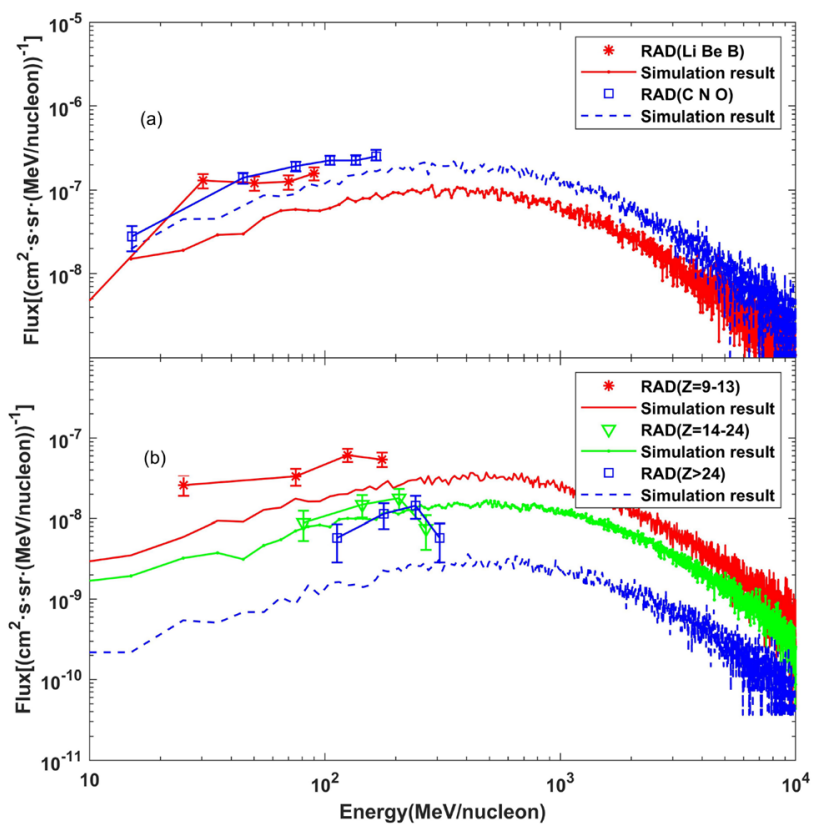

Fig. 4 (Color online) Simulated and measured charged particles' spectra, for zenith angles smaller than $30^{\circ}: \mathbf{a} \mathrm{Li}-\mathrm{Be}-\mathrm{B}$ and $\mathrm{C}-\mathrm{N}-\mathrm{O} ; \mathbf{b}$ particle groups $Z=9-13, Z=14-24$, and $Z>24$

sections. Based on the previous work, the cross sections for light fragments simulated by the G4QMD model were lower than the experimental data [35].

Heavy charged particles incident from the entire upper hemisphere were also recorded in the simulation, and the simulated spectra for zenith angles smaller than $90^{\circ}$ are 
plotted in Fig. 5. Compared with the spectrum within the $30^{\circ}$ zenith angle, for light particles, the flux averaged over the $90^{\circ}$ zenith angle was close to the flux for zenith angles below $30^{\circ}$. However, for heavy particles, the flux for the zenith angles below $90^{\circ}$ was slightly smaller than the flux for zenith angles below $30^{\circ}$. The spectra of electrons, muons, and pions were also calculated, owing to their significant contributions to the absorbed dose, and are plotted in Fig. 6.

\section{Radiation dose received by a typical human body}

Accurate estimation of the dose received by astronauts is an essential precondition for successful manned deep-space exploration missions. In addition, with the wide use of nuclear technology in material physics and medical physics [38-46], it is also important to be able to calculate the radiation dose received by a typical human in the corresponding radiation environment. Based on the prior simulation results, we calculated the radiation dose that would have been received by astronauts on the Mars surface.

According to the International Commission on Radiological Protection (ICRP) [47], the equivalent dose for a tissue, $H_{T}$, is defined by

$H_{T}=\sum_{R} W_{R} D_{T, R}$

where $D_{T, R}$ is the absorbed dose for tissue $T$ caused by

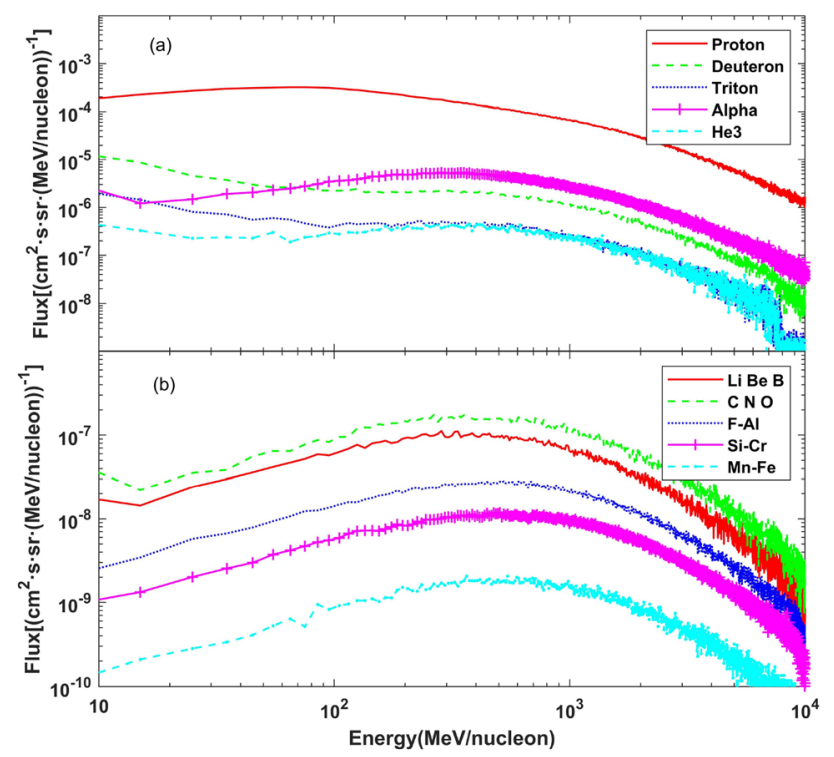

Fig. 5 (Color online) Simulated spectra of particles, for zenith angles smaller than $90^{\circ}$ : a protons, deuterons, tritons, ${ }^{3} \mathrm{He}$, and alpha particles. b $\mathrm{Li}-\mathrm{Be}-\mathrm{B}, \mathrm{C}-\mathrm{N}-\mathrm{O}, \mathrm{F}-\mathrm{Al}, \mathrm{Si}-\mathrm{Cr}$, and $\mathrm{Mn}-\mathrm{Fe}$

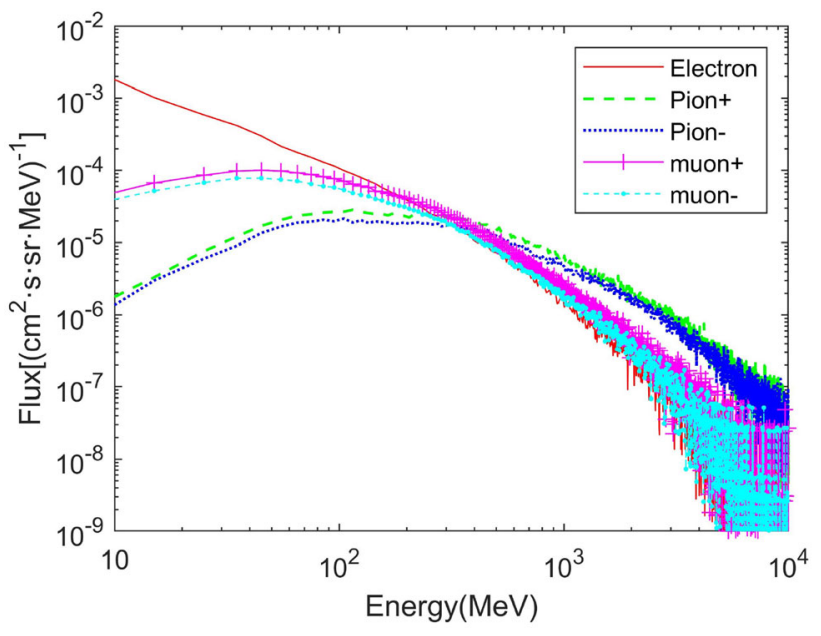

Fig. 6 (Color online) Simulated spectra of electrons, pions, and muonsm, for zenith angles smaller than $90^{\circ}$

radiation $R$, and $W_{R}$ is the radiation weighting factor for radiation $R$. The effective dose, $E$, is defined as

$E=\sum_{T} W_{T} H_{T}$

where $W_{T}$ is the tissue weighting factor for tissue $T$. Tables 3 and 4 list the values of $W_{T}$ and $W_{R}$, respectively. The ICRP recommends that the effective dose received during occupational exposure and in public should not exceed $20 \mathrm{mSv} / \mathrm{y}$ and $1 \mathrm{mSv} / \mathrm{y}$ [47], respectively.

\subsection{Model setup}

The geometrical simulation model is shown in Fig. 7. The human phantom model [48] was borrowed from the Geant4 advanced example "human phantom" and placed on the Mars surface. A cubical regolith $\left(10 \times 10 \times 6 \mathrm{~m}^{3}\right)$ was built, with the density of $1.7 \mathrm{~g} / \mathrm{cm}^{3}$. Then, the simulated particles' spectra within the $90^{\circ}$ zenith angle (in Figs. 5, 6) were used for simulating primary particles and projecting them onto the surface from the top of the geometrical model. For each step in the simulation, the energy deposited by different particles into different human body organs was recorded. After the simulation, the values of the

Table 3 Tissue weighting factors $W_{T}$ (extracted from Ref [47])

\begin{tabular}{lll}
\hline Tissue & $W_{T}$ & $\sum W_{T}$ \\
\hline $\begin{array}{l}\text { Bone-marrow (red), colon, lung, stomach, breast, } \\
\text { remainder tissues }\end{array}$ & 0.12 & 0.72 \\
Gonads & 0.08 & 0.08 \\
Bladder, oesophagus, liver, thyroid & 0.04 & 0.16 \\
Bone surface, brain, salivary glands, skin & 0.01 & 0.04 \\
\hline
\end{tabular}


Table 4 Radiation weighting factors $W_{R}$ (extracted from ref [47])

\begin{tabular}{ll}
\hline Radiation type $(R)$ & Radiation weighting factors $\left(W_{R}\right)$ \\
\hline Photons & 1 \\
Electrons and muons & 1 \\
Protons and charged pions & 2 \\
$\alpha$ particles, fission fragments, heavy ions & 20 \\
Neutrons & $2.5+18.2 \exp \left\{-\left[\ln \left(E_{\mathrm{n}}\right)\right]^{2} / 6\right\}, E_{\mathrm{n}}<1 \mathrm{MeV}$ \\
& $5.0+17.0 \exp \left\{-\left[\ln \left(2 E_{\mathrm{n}}\right)\right]^{2} / 6\right\}, 1 \mathrm{MeV} \leq E_{\mathrm{n}} \leq 50 \mathrm{MeV}$ \\
& $2.5+3.25 \exp \left\{-\left[\ln \left(0.04 E_{\mathrm{n}}\right)\right]^{2} / 6\right\}, E_{\mathrm{n}}>50 \mathrm{MeV}$ \\
\hline
\end{tabular}

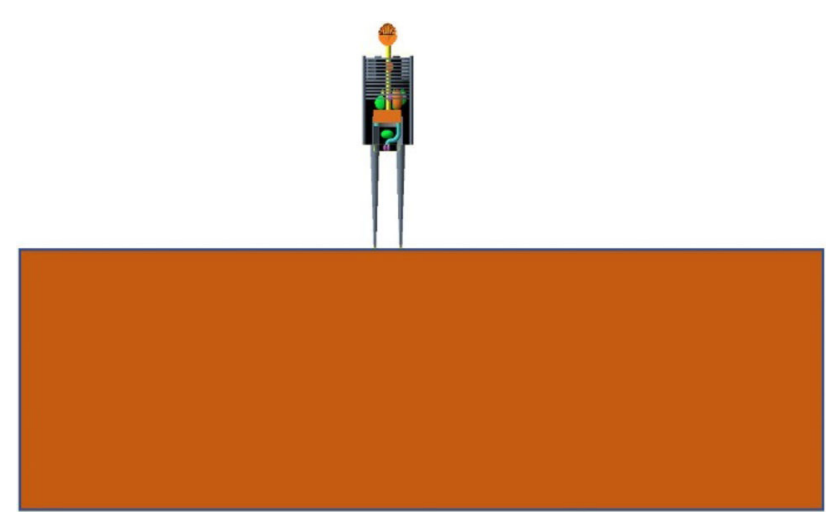

Fig. 7 (Color online) Geometrical simulation model

total deposited energy, the absorbed dose, and the equivalent dose delivered by different particles to different organs were calculated. In the simulation, the total sampling number of each primary particle was set to 5 million. For each scenario, the simulation was repeated six times, and the uncertainties shown in this paper represent, in each case, one standard deviation from the corresponding mean.

\subsection{Results}

Figure 8 shows the radiation dose received by different human body organs, for a typical human staying on the Mars surface for one year. The distribution of the deposited energies in the body organs is plotted in Fig. 8a. Evidently, the energy deposited in the trunk was the highest, reaching $(9.05 \pm 0.04) \times 10^{12} \mathrm{MeV} / \mathrm{y}$. The body part receiving the second highest amount of the radiation energy were legs, with the deposited energy per leg reaching $(2.02 \pm 0.02) \times 10^{12} \mathrm{MeV} / \mathrm{y}$. This large deposition occurred because the mass and volume of the trunk and legs were larger than those of the other body parts. The amount of energy deposited in the head and skull was also high; however, the amounts of energy deposited in smaller organs, such as left/right adrenal and thyroid glands, were among the lowest. Figure $8 \mathrm{~b}$ shows the simulated absorbed dose distribution across the different body parts. The distribution of the absorbed dose differs significantly from the distribution of deposited energy. The absorbed dose was the highest for the brain, reaching $62.0 \pm 1.7 \mathrm{mGy} / \mathrm{y}$, followed by the thyroid and skull. The absorbed doses for the thymus, upper spine, left/right lung, and left/right clavicle were also relatively high; however, the absorbed doses for the lower body organs were relatively low; for example, the absorbed doses for the left/right leg and leg bone were approximately $35 \mathrm{mGy} / \mathrm{y}$. Evidently, the difference between the doses received by the different organs was significant, because primary particles were incident from above; consequently, some smaller-energy particles only affected the superficial layers of the human body. Thus, upper-body organs, such as the brain and the thyroid gland, received higher radiation doses than lower-body organs. The average absorbed dose for the entire body was $43.8 \pm 0.4 \mathrm{mGy} / \mathrm{y}$. Taking the quality of different radiation sources into account, we calculated the equivalent doses for the different organs, and the results are shown in Fig. 8c. The equivalent dose for the brain was the highest, reaching $234.1 \pm 8.0 \mathrm{mSv} / \mathrm{y}$, agreeing well with the corresponding RAD data [31]. For the skull, adrenal, and upper spine, the equivalent doses were also relatively high, all above 200 $\mathrm{mSv} / \mathrm{y}$. For the leg bone, the equivalent dose was the lowest, just $116.4 \pm 1.7 \mathrm{mSv} / \mathrm{y}$. The average equivalent dose for the entire body was approximately $153 \mathrm{mSv} / \mathrm{y}$, much lower than the corresponding RAD data. We posit that this difference could be attributed to the fact that a typical human body is much larger than the RAD detector, and because the mass distribution in the body is not uniform. The lower part of the body and inner organs received less radiation, so that the average equivalent dose for the entire body was lower than the corresponding RAD data.

To analyze the contributions of different particles to the radiation dose received by the human body, we extracted the deposited energy and the absorbed dose in the human body owing to electrons, photons, pions, muons, protons, $\alpha$ particles, and heavy ions $(Z \geq 3)$, respectively. The results are plotted in Fig. 9, except for photons, because their contribution was far less than those of other particles. The energy deposited by protons was $(1.08 \pm 0.01) \times 10^{13} \mathrm{MeV} /$ 


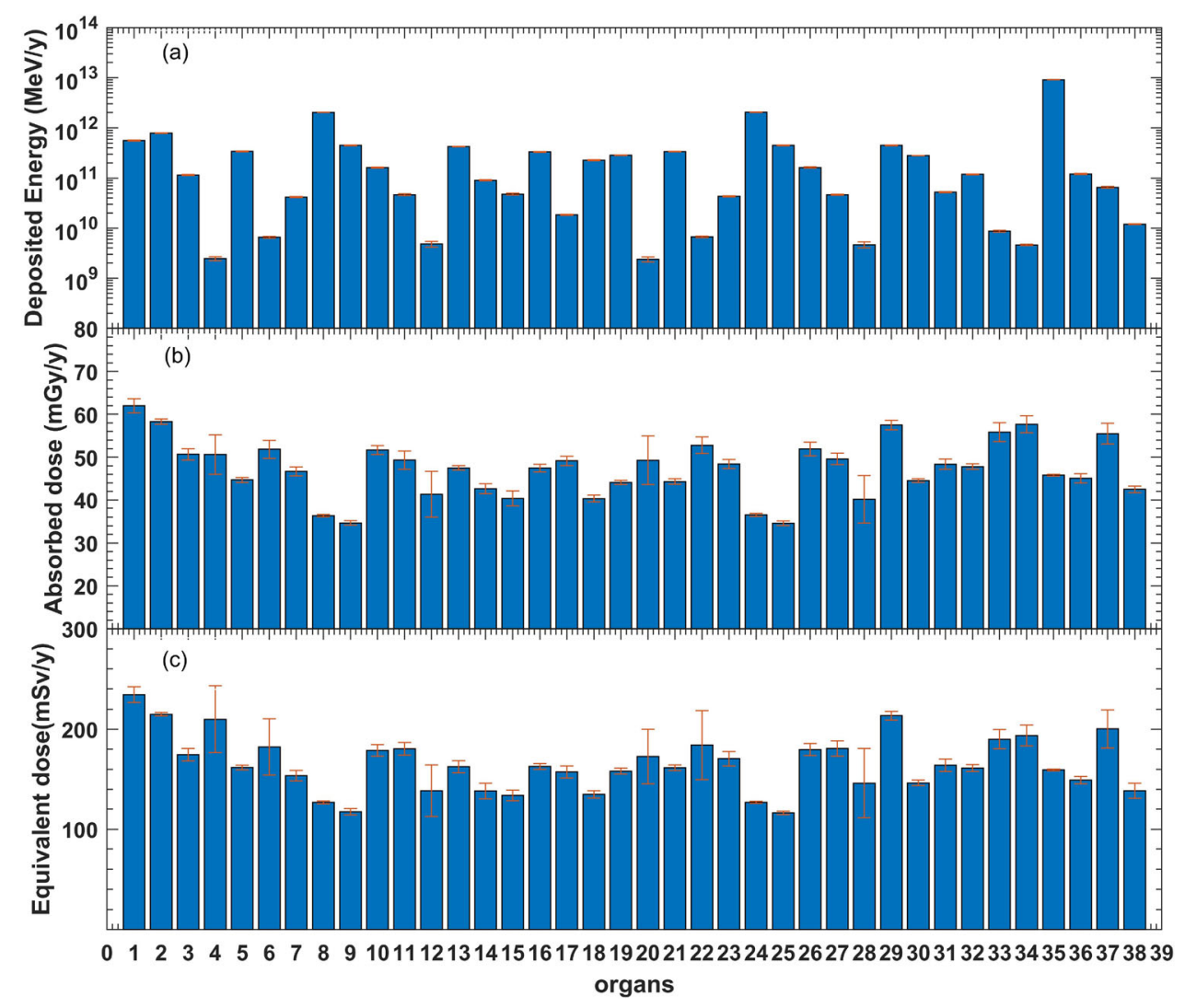

Fig. 8 (Color online) Radiation received by a human body on the Mars surface: a distribution of the radiation energy deposition in the body organs, $\mathbf{b}$ distribution of the absorbed dose in the body organs, c distribution of the equivalent dose in the body organs. The horizontal coordinate represents different organs: 1-Brain, 2-Head, 3-Heart, 4-LeftAdrenal, 5-LeftArmBone, 6-LeftClavicle, 7-LeftKidney, 8-LeftLeg, 9-LeftLegBone, 10-LeftLung, 11-LeftScapula,

$\mathrm{y}$, accounting for $56.1 \%$ of the total energy deposited in the human body. The second largest contribution was that of electrons; the corresponding deposited energy accounted for $22.6 \%$ of the total energy deposited in the body. The energies depositions by $\alpha$ particles, heavy ions, pions, and muons were smaller, accounting for $6.1 \%, 3.6 \%, 6.6 \%$, and $3.9 \%$ of the total deposited energy, respectively. The contribution of neutrons to the total deposited energy was only $0.07 \%$, much lower than that of protons. This was because the reaction cross section of neutrons with matter was very small, and most of the deposited energy was owing to secondary particles. The contributions of the different particles to the absorbed dose were similar to those for the deposited energy. However, for the equivalent dose, the situation was different. $\alpha$ particles contributed mostly to the total equivalent dose, which reached $53.7 \pm$ $1.3 \mathrm{mSv} / \mathrm{y}$, accounting for $35.1 \%$ of the total equivalent dose. The equivalent doses owing to protons and heavy ions were $49.1 \pm 0.6 \mathrm{mSv} / \mathrm{y}$ and $31.9 \pm 0.7 \mathrm{mSv} / \mathrm{y}$,
12-LeftTeste, 13-Liver, 14-LowerLargeIntestine, 15-MaleGenitalia, 16-MiddleLowerSpine, 17-Pancreas, 18-Pelvis, 19-RibCage, 20-RightAdrenal, 21-RightArmBone, 22-RightClavicle, 23-RightKidney, 24-RightLeg, 25-RightLegBone, 26-RightLung, 27-RightScapula, 28-RightTeste, 29-Skull, 30-SmallIntestine, 31-Spleen, 32-Stomach, 33-Thymus, 34-Thyroid, 35-Trunk, 36-UpperLargeIntestine, 37-UpperSpine, 38-UrinaryBladder

accounting for $32.1 \%$ and $20.9 \%$ of the total equivalent dose, respectively. The contributions of electrons, pions, muons, and neutrons were slightly smaller, accounting for only $6.5 \%, 3.8 \%, 1.4 \%$, and $0.24 \%$ of the total equivalent dose, respectively. Although the deposited energies owing to $\alpha$ particles and heavy ions were not very high, they were the main contributors to the equivalent dose. This was because the radiation weighting factors of $\alpha$ particles and heavy ions were much larger than those of the other particles. Therefore, it is necessary to consider heavy ions in GCRs, when designing future manned deep-space exploration missions.

It can be observed from Figs. 2, 3 and 4 that the simulated spectra of deuterons, tritons, and ${ }^{3} \mathrm{He}$ were much lower than those of the measured data. To assess the effect of the differences on the calculated doses to the human body, we multiplied the spectra by a scaling factor, for matching with the measured data, and recalculated the dose. The absorbed dose and the dose equivalents for the 
Fig. 9 (Color online) Contributions of different particles to the radiation dose received by the human body

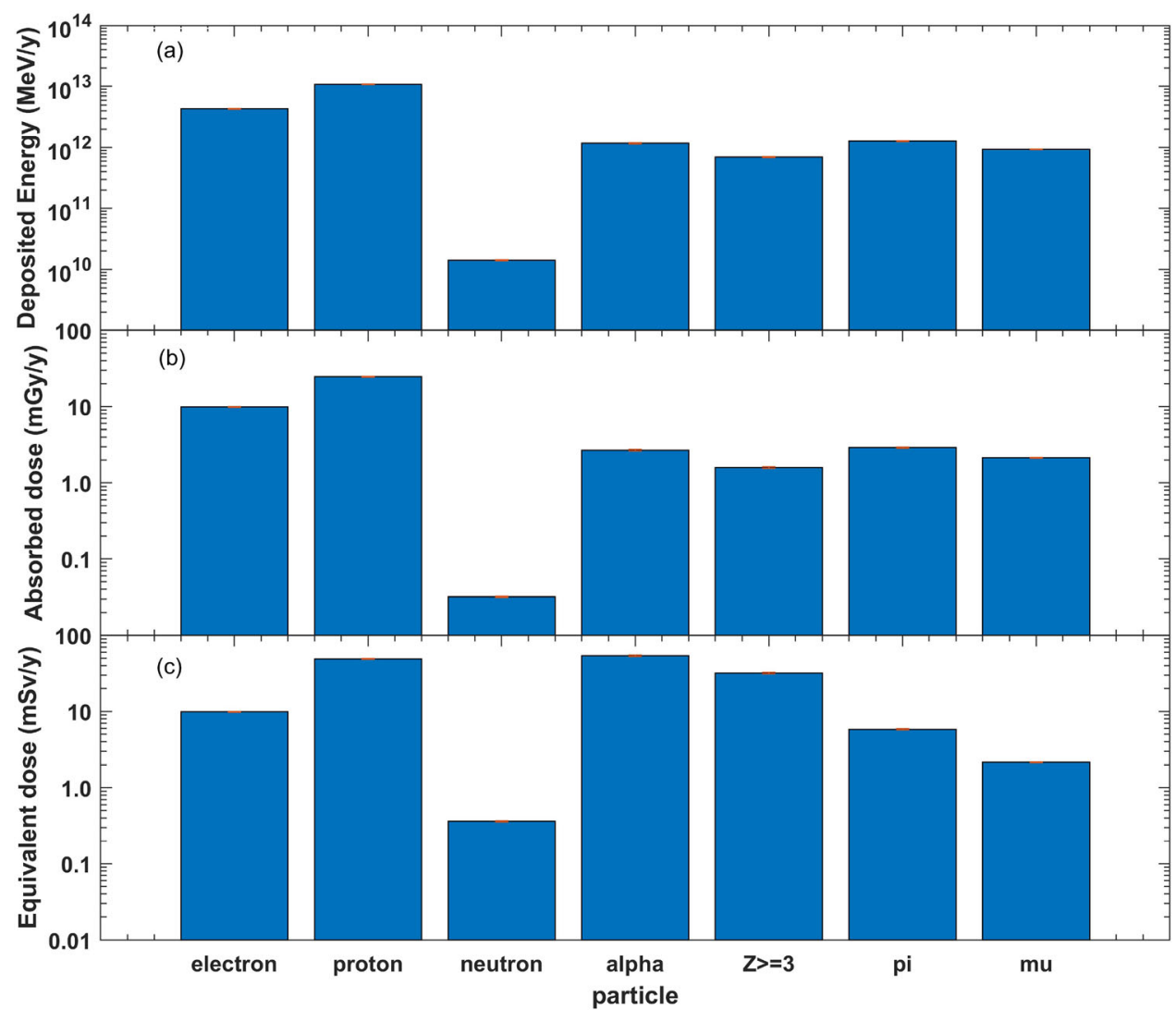

body organs, based on the scaled spectra, increased by approximately $22 \%$ on average, compared with the previous results. The average absorbed dose and the dose equivalent for the human body were $54.0 \mathrm{mGy} / \mathrm{y}$ and 191.6 $\mathrm{mSv} / \mathrm{y}$, respectively. The results show that these particles strongly affect the dose calculation, and the models in the Geant4 package should be improved for better describing nucleus-nucleus interactions.

\section{The shielding effect of the Martian soil}

The simulation results suggest that the dose that would have been received by astronauts on the Mars surface is much higher than that received on the Earth surface. Therefore, appropriate radiation protection measures are necessary. When astronauts will carry out long-term exploration missions on the Mars surface, it is likely that they will use Martian soil to build shelters, for protecting themselves from radiation. Thus, the shielding effect of Martian soil is worth investigating.

\subsection{Model setup}

A hollow cube $\left(5 \times 5 \times 3 \mathrm{~m}^{3}\right)$ was built and placed in the Mars soil, and the human phantom was placed in the cube. The thickness of the soil above the human phantom, denoted by $\mathrm{d}$, was adjustable. In the simulation, we considered 9 levels of the soil thickness, namely $d=0.1 \mathrm{~m}$, $0.3 \mathrm{~m}, 0.5 \mathrm{~m}, 0.7 \mathrm{~m}, 1.0 \mathrm{~m}, 1.5 \mathrm{~m}, 2.0 \mathrm{~m}, 2.5 \mathrm{~m}$, and $3 \mathrm{~m}$; for each one of these scenarios we computed the dose received by the human body. The primary particles were projected from the top of the soil according to the simulated particles' spectra on the Mars surface. The tissue energy deposition owing to different particles was recorded.

\subsection{Results}

The radiation doses on the human body at different depths under the Mars surface are plotted in Fig. 10. The deposited energy and dose rate on the Mars surface were also included for comparison. Evidently, with increasing the soil depth, the deposited energy, absorbed dose, and equivalent dose in the organs all decreased rapidly. At the depths of $0.1 \mathrm{~m}, 0.5 \mathrm{~m}$, and $1.0 \mathrm{~m}$, the absorbed dose was lower by $7.6 \%, 41.3 \%$, and $71.1 \%$, respectively, compared with the dose on the Mars surface. The reduction rate of the absorbed dose decreased as the soil thickness continued to increase. As shown in Fig. 10c, the equivalent dose in the organs dropped faster than the absorbed dose at small 


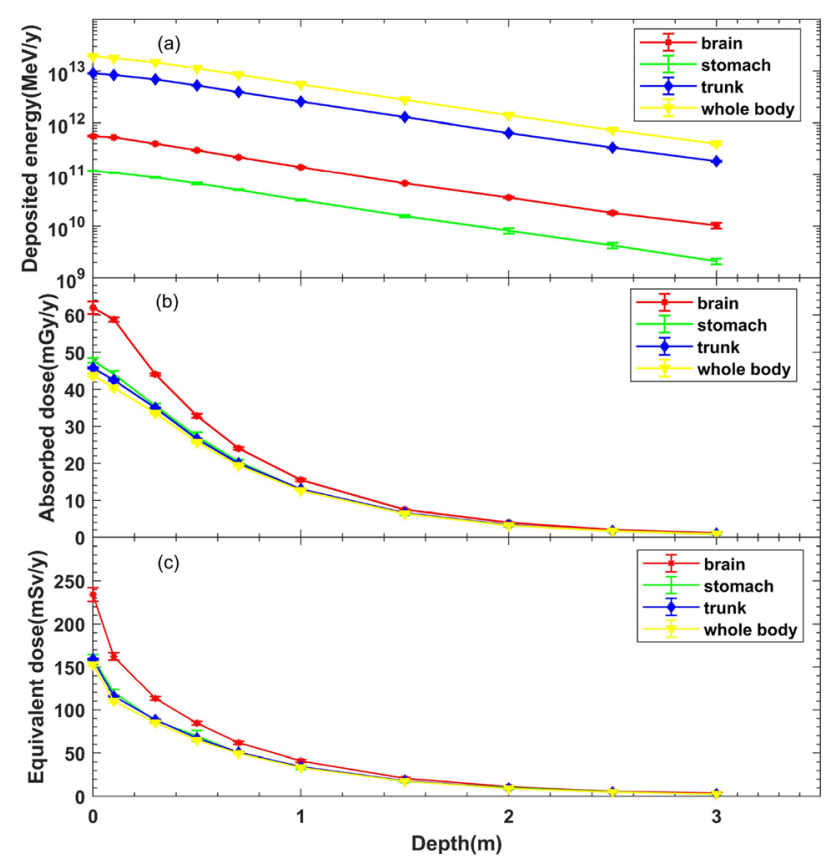

Fig. 10 (Color online) The dose on the human body, at different depths under the Mars surface

depths. For example, at the depth of $0.1 \mathrm{~m}$, the equivalent doses on the brain and whole body decreased by $30.6 \%$ and $28.1 \%$, respectively. We attribute this to the fact that energetic heavy ions collided with the Mars soil and broke into lighter fragments, with smaller radiation weighting factors. The variation of the effective dose with the soil depth was similar to that of the dose equivalent and at the soil depths of $0.1 \mathrm{~m}, 0.5 \mathrm{~m}$, and $1.0 \mathrm{~m}$, the effective dose on the entire body was $121.0 \pm 9.7 \mathrm{mSv} / \mathrm{y}, 67.6 \pm 7.9 \mathrm{mSv} / \mathrm{y}$, and $35.0 \pm 2.4 \mathrm{mSv} / \mathrm{y}$, respectively. At the depth of $1.5 \mathrm{~m}$, the average equivalent dose and effective dose on the entire body were $17.4 \pm 0.5 \mathrm{mSv} / \mathrm{y}$ and $17.9 \pm 2.4 \mathrm{mSv} / \mathrm{y}$, respectively, lower than the dose limit of $20 \mathrm{mSv} / \mathrm{y}$ for occupational exposure. At the depth of $3 \mathrm{~m}$, the equivalent doses received by different organs were between $2.0 \mathrm{mSv} / \mathrm{y}$ and $3.8 \mathrm{mSv} / \mathrm{y}$, and the effective dose on the entire body was $2.7 \pm 1.0 \mathrm{mSv} / \mathrm{y}$, still higher than the dose limit for public exposure. To confine the effective dose below 1 $\mathrm{mSv} / \mathrm{y}$, the thickness of the soil needs to be larger than $3 \mathrm{~m}$.

\section{Conclusion}

In this study, we first simulated the radiation environment on the Mars surface using the Geant4 toolkit. In the simulation, a simple geometrical model and G4QMD physics model were used. The simulation results for neutrons, photons, protons, $\alpha$ particles, and particle groups $Z=6-8, Z=9-13$, and $Z=14-24$ were in a reasonable agreement with the corresponding RAD data, with average deviations of $60.8 \%, 56.0 \%, 27.7 \%, 37.9 \%, 40.9 \%, 60.7 \%$, and $40.1 \%$, respectively. However, for deuterons, tritons, and ${ }^{3} \mathrm{He}$, which are pure secondary particles, the simulation results were much smaller than the corresponding RAD data, which might have been owing to the underestimation of the production cross sections in the G4QMD model. In addition, the particles' spectrum from the entire upper hemisphere was also obtained.

Based on the simulated radiation environment, we calculated the radiation dose received by the human body on the Mars surface. The results showed that the doses absorbed by different organs differed significantly from each other. The absorbed doses for the brain, thyroid, and skull were relatively high, but the doses for the legs and male genitalia were much lower. The average absorbed dose and equivalent dose of the entire body were $43.8 \pm$ $0.4 \mathrm{mGy} / \mathrm{y}$ and $152.9 \pm 2.0 \mathrm{mSv} / \mathrm{y}$, respectively. Further analysis showed that most of the dose was induced by $\alpha$ particles, protons, and heavy ions. The shielding effect of the Mars soil on radiation was also studied. With increasing soil depth, the deposited energy, absorbed dose, and equivalent dose for the organs decreased rapidly. At the depth of $1.5 \mathrm{~m}$, the effective dose for the entire body was $17.9 \pm 2.4 \mathrm{mSv} / \mathrm{y}$, lower than the dose limit recommended by the ICRP for occupational exposure. However, for confining the effective dose to the safe range for public exposure, the soil thickness should exceed $3 \mathrm{~m}$.

Open Access This article is licensed under a Creative Commons Attribution 4.0 International License, which permits use, sharing, adaptation, distribution and reproduction in any medium or format, as long as you give appropriate credit to the original author(s) and the source, provide a link to the Creative Commons licence, and indicate if changes were made. The images or other third party material in this article are included in the article's Creative Commons licence, unless indicated otherwise in a credit line to the material. If material is not included in the article's Creative Commons licence and your intended use is not permitted by statutory regulation or exceeds the permitted use, you will need to obtain permission directly from the copyright holder. To view a copy of this licence, visit http://creativecommons. org/licenses/by/4.0/.

Author Contributions All authors contributed to the study conception and design. Material preparation, data collection and analysis were performed by Jun-Liang Chen, Su-Jun Yun, Tie-Kuang Dong, Zhong-Zhou Ren and Xiao-Ping Zhang. The first draft of the manuscript was written by Jun-Liang Chen and all authors commented on previous versions of the manuscript. All authors read and approved the final manuscript.

\section{References}

1. G.B. Yu, E.H. Liu, G.L. Liu et al., Moderate resolution imaging camera (MoRIC) of China's first mars mission Tianwen-1. Earth Planet. Phys. 4(4), 364-370 (2020). https://doi.org/10.26464/ epp2020056 
2. M. Durante, Space radiation protection: destination mars. Life Sci. Space Res. (Amst) 1, 2-9 (2014). https://doi.org/10.1016/j. 1ssr.2014.01.002

3. F.A. Cucinotta, M. Durante, Cancer risk from exposure to galactic cosmic rays: implications for space exploration by human beings. Lancet Oncol. 7, 431-435 (2006). https://doi.org/ 10.1016/S1470-2045(06)70695-7

4. N.A. Schwadron, L. Townsend, K. Kozarev et al., Earth-moonmars radiation environment module framework. Space Weather 8, S00E02 (2010). https://doi.org/10.1029/2009SW000523

5. Á. Vicente-Retortillo, F. Valero, L. Vázquez et al., A model to calculate solar radiation fluxes on the Martian surface. J. Space Weather Space Clim. 5, A33 (2015). https://doi.org/10.1051/ swsc/2015035

6. F.F. Badavi, D.O. Adams, J.W. Wilson, On the validity of the aluminum equivalent approximation in space radiation shielding applications. Adv. Space Res. 46, 719-727 (2010). https://doi. org/10.1016/j.asr.2010.04.006

7. M. Durante, F.A. Cucinotta, Physical basis of radiation protection in space travel. Rev. Mod. Phys. 83, 1245-1281 (2011). https:// doi.org/10.1103/RevModPhys.83.1245

8. M. Vuolo, G. Baiocco, S. Barbieri et al., Exploring innovative radiation shielding approaches in space: a material and design study for a wearable radiation protection spacesuit. Life Sci. Space Res. (Amst) 15, 69-78 (2017). https://doi.org/10.1016/j. 1ssr.2017.08.003

9. A.I. Mrigakshi, D. Matthiä, T. Berger et al., How Galactic Cosmic Ray models affect the estimation of radiation exposure in space. Adv. Space Res. 51, 825-834 (2013). https://doi.org/10. 1016/j.asr.2012.10.017

10. D. Matthiä, T. Berger, A.I. Mrigakshi et al., A ready-to-use galactic cosmic ray model. Adv. Space Res. 51, 329-338 (2013). https://doi.org/10.1016/j.asr.2012.09.022

11. B. Ehresmann, C.J. Zeitlin, D.M. Hassler et al., The charged particle radiation environment on Mars measured by MSL/RAD from November 15, 2015 to January 15, 2016. Life Sci. Space Res. (Amst) 14, 3-11 (2017). https://doi.org/10.1016/j.lssr.2017. 07.004

12. T. Sato, K. Niita, V.A. Shurshakov et al., Evaluation of dose rate reduction in a spacecraft compartment due to additional water shield. Cosm. Res. 49, 319-324 (2011). https://doi.org/10.1134/ S0010952511040083

13. F.A. Cucinotta, S. Hu, N.A. Schwadron et al., Space radiation risk limits and Earth-Moon-Mars environmental models. Space Weather 8, S00E09 (2010). https://doi.org/10.1029/ 2010SW000572

14. S. Trovati, F. Ballarini, G. Battistoni et al., Human exposure to space radiation: role of primary and secondary particles. Radiat. Prot. Dosim. 122, 362-366 (2006). https://doi.org/10.1093/rpd/ ncl438

15. B. Ehresmann, C. Zeitlin, D.M. Hassler et al., Charged particle spectra obtained with the Mars Science Laboratory Radiation Assessment Detector (MSL/RAD) on the surface of Mars. J. Geophys. Res. Planets 119, 468-479 (2014). https://doi.org/10. 1002/2013JE004547

16. L. de la Fuente Rosales, S. Incerti, Z. Francis et al., Accounting for radiation-induced indirect damage on DNA with the Geant 4-DNA code. Phys. Med. 51, 108-116 (2018). https://doi.org/10. 1016/j.ejmp.2017.09.048

17. N. Lampe, M. Karamitros, V. Breton et al., Mechanistic DNA damage simulations in Geant4-DNA part 1: a parameter study in a simplified geometry. Phys. Med. 48, 135-145 (2018). https:// doi.org/10.1016/j.ejmp.2018.02.011

18. J. Chen, S. Yun, T. Dong et al., Investigate the radiation-induced damage on an atomistic DNA model by using Geant4-DNA toolkit. Nucl. Instrum. Methods Sect. B 494-495, 59-67 (2021). https://doi.org/10.1016/j.nimb.2021.03.010

19. J.C. Chancellor, G.B. Scott, J.P. Sutton, Space radiation: the number one risk to astronaut health beyond low earth orbit. Life (Basel) 4, 491-510 (2014). https://doi.org/10.3390/life4030491

20. T.C. Slaba, N.N. Stoffle, Evaluation of HZETRN on the Martian surface: sensitivity tests and model results. Life Sci. Space Res. (Amst) 14, 29-35 (2017). https://doi.org/10.1016/j.lssr.2017.03. 001

21. D. Matthiä, B. Ehresmann, H. Lohf et al., The Martian surface radiation environment - a comparison of models and MSL/RAD measurements. J. Space Weather Space Clim. 6, A13 (2016). https://doi.org/10.1051/swsc/2016008

22. W.C. de Wet, L.W. Townsend, A calculation of the radiation environment on the Martian surface. Life Sci. Space Res. (Amst) 14, 51-56 (2017). https://doi.org/10.1016/j.1ssr.2017.07.008

23. J.W. Norbury, T.C. Slaba, N. Sobolevsky et al., Comparing HZETRN, SHIELD, FLUKA and GEANT transport codes. Life Sci. Space Res. (Amst) 14, 64-73 (2017). https://doi.org/10.1016/ j.1ssr.2017.04.001

24. J. Flores-McLaughlin, Radiation transport simulation of the Martian GCR surface flux and dose estimation using spherical geometry in PHITS compared to MSL-RAD measurements. Life Sci. Space Res. (Amst) 14, 36-42 (2017). https://doi.org/10.1016/ j.1ssr.2017.07.007

25. D. Matthiä, D.M. Hassler, W. de Wet et al., The radiation environment on the surface of Mars-Summary of model calculations and comparison to RAD data. Life Sci. Space Res. (Amst) 14, 18-28 (2017). https://doi.org/10.1016/j.1ssr.2017.06.003

26. H.N. Ratliff, M.B.R. Smith, L. Heilbronn, Simulation of the GCR spectrum in the Mars curiosity rover's RAD detector using MCNP6. Life Sci. Space Res. (Amst) 14, 43-50 (2017). https:// doi.org/10.1016/j.lssr.2017.07.003

27. D. Matthiä, T. Berger, The radiation environment on the surface of Mars-Numerical calculations of the galactic component with GEANT4/PLANETOCOSMICS. Life Sci. Space Res. 14, 57-63 (2017). https://doi.org/10.1016/j.1ssr.2017.03.005

28. S. Agostinelli, J. Allison, K. Amako et al., Geant4-a simulation toolkit. Nucl. Instrum. Methods Sect. A 506, 250-303 (2003). https://doi.org/10.1016/S0168-9002(03)01368-8

29. J. Allison, K. Amako, J. Apostolakis et al., Geant 4 developments and applications. IEEE Trans. Nucl. Sci. 53, 270-278 (2006). https://doi.org/10.1109/TNS.2006.869826

30. J. Allison, K. Amako, J. Apostolakis et al., Recent developments in Geant4. Nucl. Instrum. Methods Sect. A 835, 186-225 (2016). https://doi.org/10.1016/j.anucene.2014.08.021

31. D.M. Hassler, C. Zeitlin, R.F. Wimmer-Schweingruber et al., Mars' surface radiation environment measured with the Mars science laboratory's curiosity rover. Science 343, 1244797 (2014). https://doi.org/10.1126/science.1244797

32. C.G. Justus, D.L. Johnson, Mars Global Reference Atmospheric Model 2001 Version (Mars-GRAM 2001) Users Guide, Marshall Space Flight Center, NASA/TM-2001-210961 (2001)

33. H. Burkhardt, V.M. Grichine, P. Gumplinger et al., Geant4 standard electromagnetic package for HEP applications. IEEE Symp. Conf. Rec. Nucl. Sci. 2004(3), 1907-1910 (2004). https:// doi.org/10.1109/NSSMIC.2004.1462617

34. A. Schumann, J. Petzoldt, P. Dendooven et al., Simulation and experimental verification of prompt gamma-ray emissions during proton irradiation. Phys. Med. Biol. 60, 4197-4207 (2015). https://doi.org/10.1088/0031-9155/60/10/4197

35. J. Chen, S. Yun, T. Dong et al., Validation of Geant 4 physics models for nuclear beams in extended media. Nucl. Instrum. Methods Sect. B 434, 113-119 (2018). https://doi.org/10.1016/j. nimb.2018.08.022 
36. Physics Reference Manual.https://geant4-userdoc.web.cern.ch/ UsersGuides/PhysicsReferenceManual/html/index.html

37. J. Köhler, C. Zeitlin, B. Ehresmann et al., Measurements of the neutron spectrum on the Martian surface with MSL/RAD. J. Geophys. Res. Planets 119, 594-603 (2014). https://doi.org/10. 1002/2013JE004539

38. W.M. Sun, N. Du, W.D. Tian et al., Secondary and activated $\mathrm{X}(\gamma)$ radiation of SPHIC particle therapy facility. Nucl. Sci. Tech. 32(4), 1-8 (2021). https://doi.org/10.1007/s41365-021-00870-6

39. C.Y. Li, X.B. Xia, J. Cai et al., Radiation dose distribution of liquid fueled thorium molten salt reactor. Nucl. Sci. Tech. 32, 22 (2021). https://doi.org/10.1007/s41365-021-00857-3

40. C.M. Niu, M.H. Li, J.R. Dai, Cage-like radiotherapy system for noncoplanar radiotherapy. Nucl. Sci. Tech. 32, 12 (2021). https:// doi.org/10.1007/s41365-021-00848-4

41. P. Huang, S.Q. Li, R.D. Yang et al., Photon energy response optimization using few-channel spectroscopy dose method for $\mathrm{Si}$ PIN photodetector applied in personal dose equivalent measurements. Nucl. Sci. Tech. 31, 36 (2020). https://doi.org/10.1007/ s41365-020-0748-2

42. X.J. Ding, M. Yu, X. Zheng et al., Stability study of Disperse Blue 79 under ionizing radiation. Nucl. Sci. Tech. 31, 21 (2020). https://doi.org/10.1007/s41365-020-0724-x
43. Z. Ahmadi Ganjeh, M. Eslami-Kalantari, A.A. Mowlavi, Dosimetry calculations of involved and noninvolved organs in proton therapy of liver cancer: a simulation study. Nucl. Sci. Tech. 30, 173 (2019). https://doi.org/10.1007/s41365-019-0698-8

44. C.Q. Chen, X.B. Xia, Z.H. Zhang et al., Radiological environmental impact analysis of a 2-MW thorium molten salt reactor during an accident. Nucl. Sci. Tech. 30, 78 (2019). https://doi.org/ 10.1007/s41365-019-0605-3

45. S. Abdullahi, A.F. Ismail, S. Mohd Fadzil et al., Assessment of the long-term possible radiological risk from the use of ceramic tiles in Malaysia. Nucl. Sci. Tech. 30, 19 (2019). https://doi.org/ 10.1007/s41365-019-0558-6

46. Y.H. Wang, Q. Li, L. Chen et al., Simulation study of the dose and energy responses of FNTD personal neutron dosimetry. Nucl. Sci. Tech. 30, 32 (2019). https://doi.org/10.1007/s41365-0190546-x

47. A.D. Wrixon, New ICRP recommendations. J. Radiol. Prot. 28, 161-168 (2008). https://doi.org/10.1088/0952-4746/28/2/r02

48. W.S. Snyder, H.L. Fisher Jr., M.R. Ford et al., Estimates of absorbed fractions for monoenergetic photon sources uniformly distributed in various organs of a heterogeneous phantom. J. Nucl. Med. 10(Suppl 3), 7-52 (1969) 